\title{
A RESILIENT APPROACH TO INTEGRATED WATER RESOURCES MANAGEMENT IN WATER SCARCE BASINS
}

\author{
F. Yazdandoost ${ }^{*}$ and S. Moradian
}

Department of Civil Engineering, K N Toosi University of Technology, Tehran, Iran

Published online: 15 May 2016

\begin{abstract}
Resistance is defined as the ability of a system to withstand a disturbance without any reaction, and resilience as the ability of a system to recover easily from a reaction to a disturbance. These concepts are often applied to risk management by adopting a systems approach. The system may be defined as the socio-economic and physical characteristics of the basin where disturbances may affect the normal socio-economic and natural functioning of society and ecosystems. To make the resilience concept more tangible, indicators are defined and to quantify resilience, the aspects which give insight into the reaction of systems are studied and quantified. These aspects were taken as; "Amplitude": The magnitude of the reaction, "Gradualist": The increase of reactions with increasingly severe conditions and "Recovery Rate". The resilience of a system is presumed to be larger when the amplitude is smaller, the gradualist is larger or/and the recovery rate is larger. A toolbox has been developed to investigate the role of resilient risk management on decision/policy making in semi-arid basins facing water scarcity. The toolbox comprises an allocation model, namely the Water Evaluation and Planning System (WEAP) model, as the central engine where water management scenarios and the effect of proposed developments on water resources may be investigated in an integrated manner based on sustainability criteria encompassing socioeconomic and environmental characteristics of the basin.
\end{abstract}

\footnotetext{
Author Correspondence, e-mail: yazdandoost@kntu.ac.ir

doi: http://dx.doi.org/10.4314/jfas.v8i3s.172
} 
The toolbox has been utilized for the case of Lake Urmia basin. Lake Urmia, one of the largest saltwater lakes on earth and a highly endangered ecosystem, is on the brink of desiccation. Considering no significant trend in the drought pattern, Lake Urmia's observed physiographic changes may be attributed to the adverse effects of unsustainable development plans and excessive irrigation projects in the basin. To combat this potential catastrophe, the authorities have taken rapid action in drastically curbing dam construction and utilisation and to reduce the overall agricultural allocation by $40 \%$ in Lake Urmia basin. The results obtained from the proposed toolbox indicates that adopting a resilient risk management approach would imply that, for instance for the critically important issue of agriculture with possible grave social implications, the same outcomes for the lake's water demand may be reached through far less reduction.

Keywords: Resilience; Resilient risk management; Water scarce basin; Lakes.

\section{INTRODUCTION}

Water shortage has often been the source of competitions and conflicts amongst stakeholders. Integrated Water Resources Management (IWRM) is seen worldwide as appropriate means of conflict resolution. IWRM is the response to the growing pressure on water resources systems as a result of growing population and socio-economic developments. Water resources management has undergone a drastic change world-wide, moving from a mainly supplyoriented, engineering biased approach towards a demand-oriented, multi-sectoral approach, often labelled as Integrated Water Resources Management. The concept of IWRM lets us move away from 'water master planning', which focuses on water availability and development, towards 'comprehensive water policy planning' which addresses the interaction between different sub-sectors, seeks to establish priorities, considers institutional requirements, and deals with the building of capacity. IWRM should not be seen as a 'model' that has to be enforced upon the system. IWRM is much more a process. According to GWP 2000, IWRM is a process which promotes the co-ordinated development and management of water, land and related resources, in order to maximize the resultant economic and social welfare in an equitable manner without compromising the sustainability of vital ecosystems. Arid and semi - arid regions are defined as areas where the rainfall patterns are inherently erratic and where, generally, precipitation is below potential evapotranspiration. Due to limited access to shallow waters, lack of freshwater resources and presence of often polluted ground water; water is at its most scarce in such areas. The hydrological regime in these areas 
is extreme and highly variable and globally, these areas face the greatest pressures to deliver and manage freshwater resources. Scarcity is further defined at basin scale through a consistent negative balance when the demand is usually higher than the supply. Despite considerable developments in risk management techniques in water scarce basins, management deficiencies persist due to numerous uncertainties. Problems are further exacerbated by population growth, increasing domestic water use, expansion of agriculture, pollution, and the threat of climate change. As a result a paradigm shift in approach is required from the conventional risk management approaches where basin security is increased in the face of water scarcity. In the proposed approach, the sustainability of a system is defined in terms of its ability to recover from a disturbance to an appropriate initial position. Resilience is therefore considered alongside resistance where the former refers to recovery from the disturbance and the latter the resistance against the disturbance. Resilience has been defined in various forms depending on its application. Resilience originally defined by Holling (1973) for ecological systems was further extended to social systems (Adger 2000), and was later analysed in social-ecological systems (Walker et al. 2002). Pimm (1984) defined resilience as the speed at which a system reovers upon a disturbance. Inspired by such definitions, various theories were presented for resilient flood management approach in river basins and lowland rivers (De Bruijn 2004, Yazdandoost and Bozorgy 2008). Some studies, however, have attempted to develop indicators to quantitatively reflect the effectiveness of pertaining measures in considering the economic-technical aspects in urban drainage risk management systems (Tahmasebi Birgani and Yazdandoost 2014, Tahmasebi Birgani et al. 2013). Karamouz (2009) defined resilience as the time taken for the system to recover from a disturbance to its normal condition. This research intends to investigate the impact of resilience on water scarce basins from an IWRM perspective.

\section{RESILIENCE IN RISK MANAGEMENT}

\section{A. Resilience}

The stability of a system is highly dependent on both resilience and resistance. There are two views to define resilience. The first view, termed "engineering resilience" focuses on system's behavior near stable equilibrium and is indicative of a system's speed of return to an equilibrium following a disturbance. Based on this assumption, resilience can be measured by resistance to disturbances and rate of return to equilibrium. The second view, concentrates on a system's behavior near the boundary of a domain of attraction far from any equilibrium 
where instabilities can flip to another domain and is a buffer capacity or ability to absorb perturbation. This is measured as magnitude of a disturbance which can be absorbed before system changes its structure by changing variables and processes that control system's behavior.

Three aspects may be inferred from the definition of the stability of system on the basis of the system's behavior in response to disturbances. These aspects comprise: the system does not show specific behavior, the system shows response and the system recovers from disturbances to the previous normal situation prior to the occurrence of disturbance. This may be suitably explained as a system's behavior under disturbances based on a response curve. When a system is affected by any disturbance, system's response is different depending on the magnitude of disturbance (Fig. 1a). As shown in Fig. 1a, for small disturbances, the system may not react at all which implies the first perspective, whereas for larger ones, the system may react with its variation amplitude proportional to the increase in disturbance, and somehow implies the second perspective. The third aspect illustrated in Fig. 1b shows that the resilient system state tends to return to the previous normal condition after reacting to disruption. It should be noted that the system recovery does not mean that the system should return to the exact same previous situation but the main characteristics of system should be restored. Accordingly, three domains can be considered in these two schematic diagrams: resistance domain, reaction domain and recovery domain while quantifying these domains may enable evaluating the stability of a system to some extent. The resistance domain refers to the condition in which system has no reaction to disruption. In other words, system can easily bear the perturbation applied to it. In reaction domain the system's behavior is different than it was in the resistance domain. This is a range in which the system cannot withstand the disturbance and will be inevitably affected by it. There are two considerable aspects in this behavior: amplitude and trend of reaction. For a resilient system, it is more desirable for the reaction amplitude to be low and reaction trend to be more gradual. In addition, recovery domain can show how a system recovers from disturbances. The rate of this recovery process is significant to understand how fast a system can attain normal performance. It therefore seems in addition to system itself and its properties, disturbances, response to disturbances and the recovery should be described to define the stability of a system (Tahmasebi Birgani et al. 2013). 


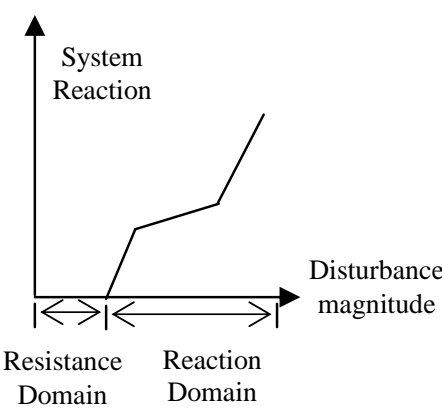

(b)

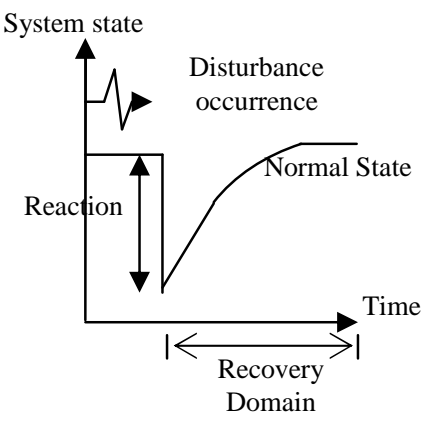

(a)

Fig.1. System's behavior in response to disturbance: (a) system's reaction to a disturbance, (b) system recovery from a disturbance to a normal state

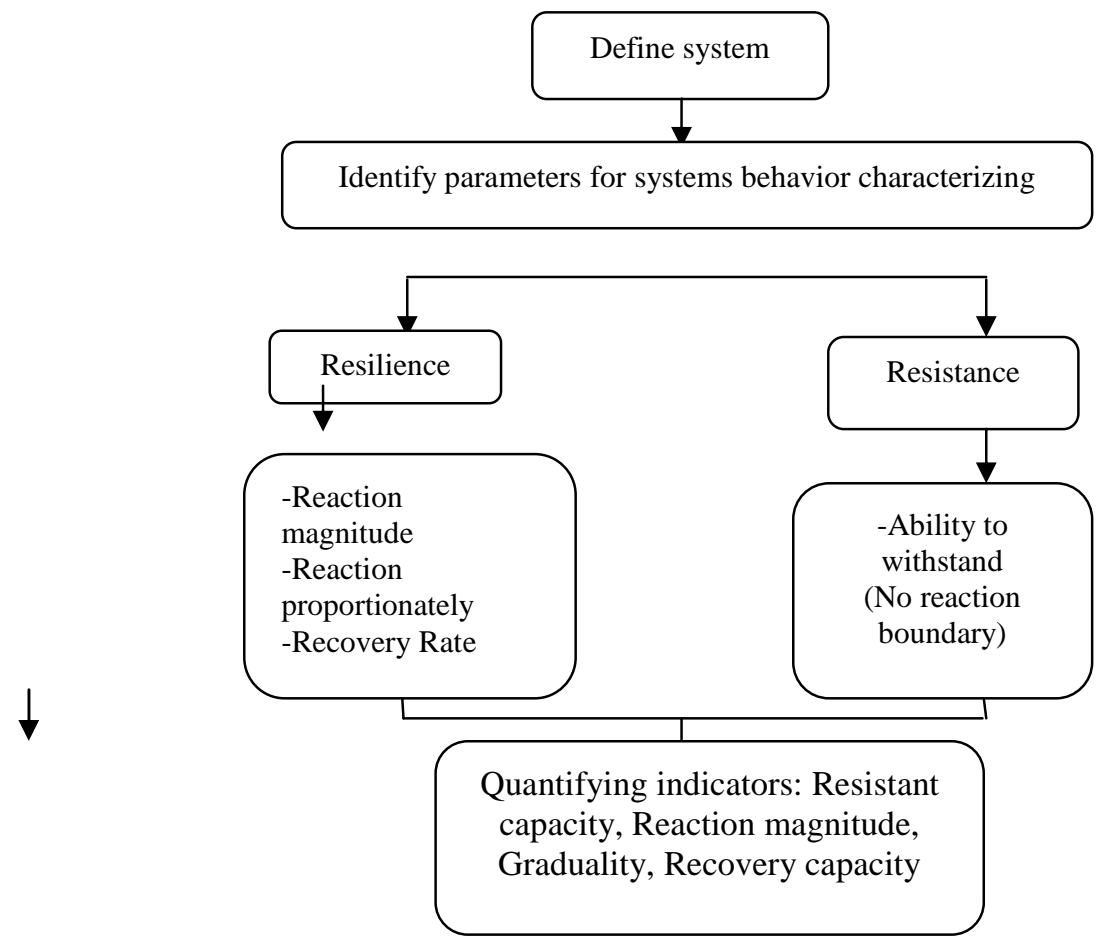

Fig.2. Steps to reach the stability of the system

One of the most important characteristics of a stable system is that the system tends to return to previous normal state of operation but not the exact previous situation as it would have operated if no disturbance had occurred. Fig. 2 summarizes the framework suggested here to evaluate the stability of a system. It is $t$ important to identify parameters by which the system's behavior in response to disturbances can be appropriately characterized. Specific 
indicators which can entirely address parameters are specified and quantified. "reaction magnitude", "graduality" and "recovery capacity" are the indicators considered to show the risk magnitude and risk reduction by the measures, the proportionality of the damages (gradual reaction) and the rate of returning to normal operation, respectively. By adopting a system approach, these indicators may be identified in water scarce basins (Tahmasebi Birgani et al. 2013).

\section{B. Proposed Approach}

Given the scarcity associated with arid and semi-arid regions all sources of water supply, both in terms of quality and quantity, have to be carefully identified. Water systems' planning is often associated with too many uncertain parameters due to ambiguities in defining objectives, forecasts and assumptions. To achieve coherence, a broad perspective is therefore required to effectively establish the relationship between certain and uncertain parameters. Identification of parameters may then be followed by setting an allocation simulation model. Identifying the role of resilience based on IWRM may then be assisted by consideration of various scenarios encompassing technical, economic, environmental and social aspects in an integrated framework. The base/reference framework for quantity and quality of water supply and demand may be deduced based on available data. A number of different tools have shown specific capabilities in simulating allocations under varying circumstances. Sequence of events envisaged logically and coherently would form the basis of scenarios anticipated for future developments of the area under study. Scenario generation may then be performed for socio-economical, political and environmental parameters and verified against criteria based on sustainable development. The Stockholm Environmental Institute's (SEI), WEAP model has been utilised here for this purpose. Figure 3 depicts an algorithmic procedure of the developed IWRM toolbox for this research. 


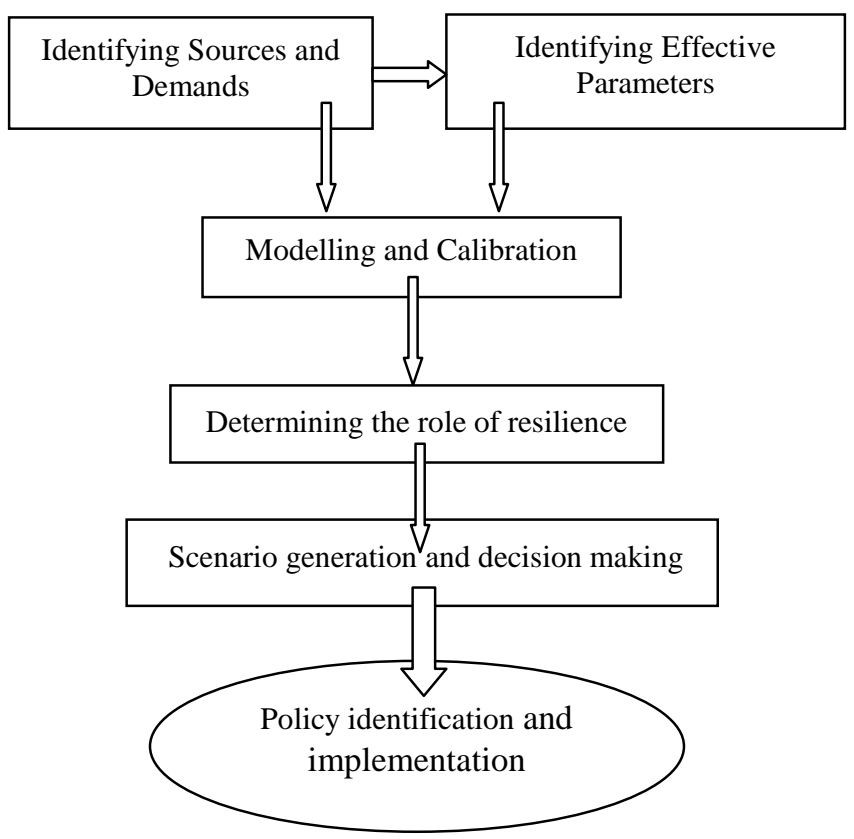

Fig.3. The proposed toolbox

\section{APPLICATION OF THE TOOLBOX}

The toolbox has been utilised for the case of Lake Urmia basin. Lake Urmia, one of the largest saltwater lakes on earth and a highly endangered ecosystem, is on the brink of desiccation. Considering no significant trend in the drought pattern, Lake Urmia's observed physiographic changes may be attributed to the adverse effects of unsustainable development plans and excessive irrigation projects in the basin.

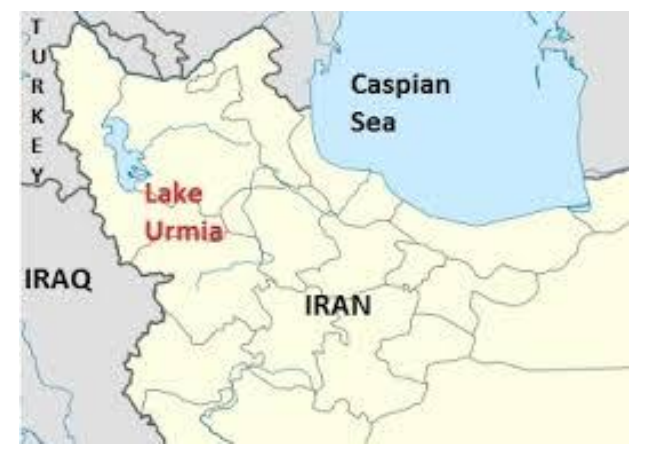

Fig.4. Urmia Lake basin

Once with a surface area of approximately half a million hectares, Lake Urmia's shoreline has been receding severely with no sign of recovery, leading to a significant shrinkage in the lake's surface area currently decreased by around $88 \%$. Lake Urmia is fed by a total of 60 (21 
seasonal or permanent, and 39 periodic) rivers with the Zarrine-Rud being the main input, reckoned to provide around $40 \%$ of the total inflow into the lake (Pirnia, 2014).

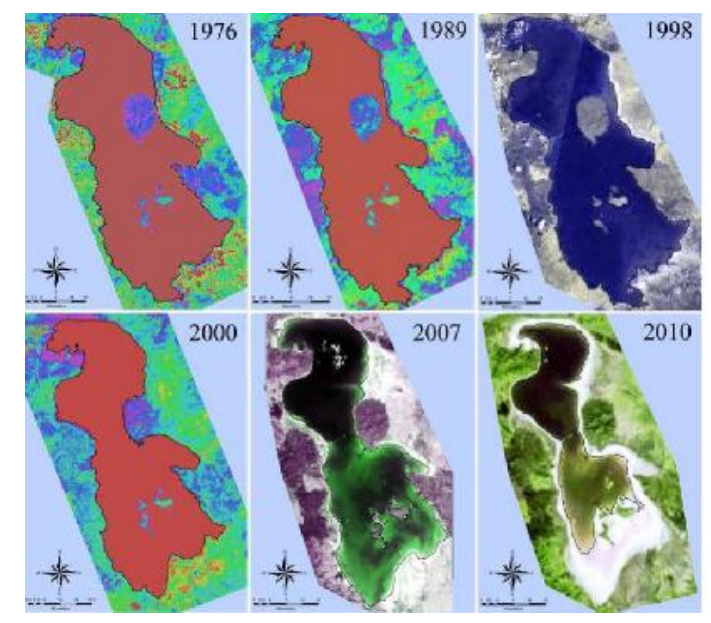

Fig.5. Lake Urmia's geophysical changes

\section{RESULTS AND DISCUSSIONS}

\section{Model setup and calibration}

Primarily all necessary data for preparation of an initial integrated model of the region were collected and classified. Given the suitable span of the existing data, year 2010 was chosen as the base year and year 2015 as the target for calibration of the model. The main demands in the study area were identified as drinking water, industry and agriculture. The Lake Urmia storage volume and the water demand were considered as criteria for calibration. System loss and returned water after consumption have not been considered for the demand where the supply requirement represents the total demand including losses and return flow. Given the importance of the lake volume in the basin, this criterion has been initially used for calibration purposes. Table 1 shows model results compared to available data for the water demand in the basin for the reference year, and table 2 shows Lake Urmia volumes over the period 2010 to 2015.

Table 1. Comparison of model results and available data for the reference year

\begin{tabular}{ccc}
\hline \multicolumn{3}{c}{ Water demand in 2010 (MCM) } \\
\hline Consumption & Model results & Available data \\
\hline Agriculture & 5549.5 & 5551.7 \\
\hline Drinking & 359.8 & 367.8 \\
\hline Industry & 88.1 & 97.3 \\
\hline
\end{tabular}


Table 2. Model results vs. available data for Lake Urmia volumes in various years (BCM)

\begin{tabular}{lllllll}
\hline Year & 2010 & 2011 & 2012 & 2013 & 2014 & 2015 \\
\hline Model resulı & 13.98( & 11.824 & 9.672 & 7.541 & 5.426 & 3.338 \\
\hline Available d $\varepsilon$ & $13.34 i$ & 11.183 & - & - & - & 2.576 \\
\hline
\end{tabular}

\section{Consideration of resilience}

In terms of water utilization, a gradual reduction of up to $40 \%$ in agricultural consumption has been introduced to alleviate the immediate problems with desiccation of Lake Urmia. The calibrated model was used to simulate gains in storage volumes of Lake Urmia as a consequence of reduction in agriculture allocation. Order of priorities were set in the model to consider drinking water as first and agriculture and industry equally as second. simulations were also projected to the year 2020. Table 3 shows the corresponding simulated results for lake volumes and basin unmet demands.

Table 3. Comparison of average lake volumes and basin unmet demands for 2015 to 2020

\begin{tabular}{lcc}
\hline \multicolumn{1}{c}{ Scenario } & $\begin{array}{l}\text { Average lake } \\
\text { volume (BCM) }\end{array}$ & $\begin{array}{l}\text { Average unmet } \\
\text { demand (BCM) }\end{array}$ \\
\hline $\begin{array}{l}\text { No reduction in } \\
\text { agriculture consumptio }\end{array}$ & 1.529 & 2.84 \\
\hline $\begin{array}{l}\text { 40\% reduction in } \\
\text { agriculture consumptio }\end{array}$ & 4.775 & 2.33 \\
\hline
\end{tabular}

To observe the effect of resilience in the toolbox approach, different indicators previously introduced, namely "recovery capacity", "Graduality" and "amplitude", may be investigated and quantified.

\section{a) Recovery rate}

The rate of recovery is proportional to the speed of recovery from the start of damages to the instant of returning to normal condition. It is rather hard to quantify this indicator as it depends upon social and physical circumstances under consideration. De brujin (2004) claimed that the three sets of effective factors determining the recovery rate may be regarded as the "physical factors", the "economic factors" and the "social factors".

Figure 6 shows different elements indicating various factors that determine the recovery rate. 


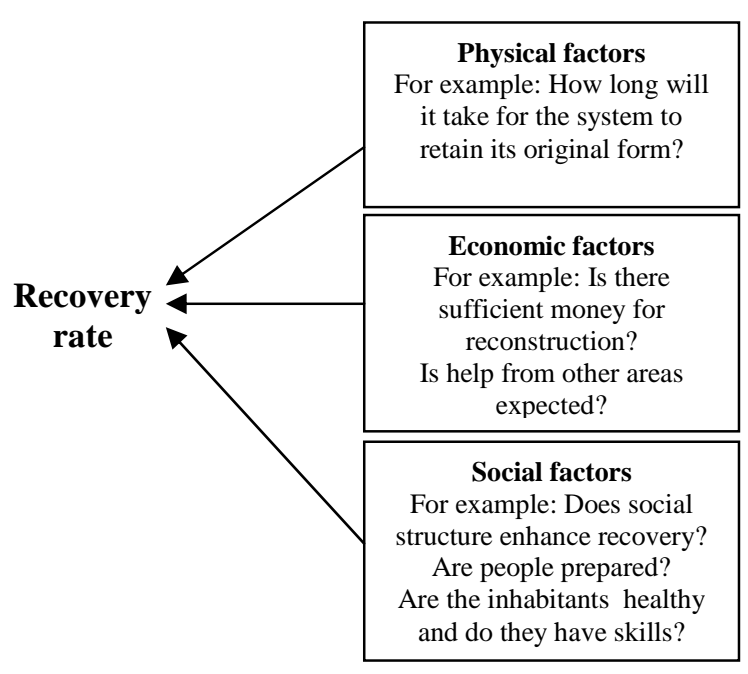

Fig.6. Factors that determine recovery capacity

In order to obtain the recovery capacity it is necessary to determine the normalised weighted average of time of disturbance over the entire basin.

$$
R D=\sum_{i=1}^{k}\left(T_{i} \times V_{i}\right) / \sum_{i=1}^{k} V_{i}
$$

where $R D$ is recovery duration of any rainfall event $(\mathrm{s}), T_{i}$ is the duration of occurance for each node (s), $V_{i}$ is the volume of water $\left(\mathrm{m}^{3}\right)$ and $\mathrm{k}$ is the number of nodes in the basin network. For representing the recovery capacity in a single number in a range between 0 and 1 , the recovery duration values should be normalised and results are then averaged over the entire range of return periods. As a result the duration of recovery should be determined for at least three different values of rainfall. The resulting value is termed as the normal duration of recovery. The normal duration of recovery may be found from:

$$
N R D_{i}=\frac{R D_{i}-R D_{\text {min }}}{R D_{\text {max }}-R D_{\text {min }}}
$$

In which, $N R D$ is the normalized recovery duration and $\mathrm{RD}_{\max }$ and $\mathrm{RD}_{\min }$ are the maximum and minimum durations of recovery respectively. Eventually the recovery capacity, R, may be found from:

$$
R=1-\frac{\sum_{i=1}^{n} N R D}{n}
$$

Where, $\mathrm{n}$ is the number of rainfall events. This relationship shows that a higher recover capacity would imply smaller low rainfall duration (De Bruijn, 2004). 


\section{b) Graduality}

Graduality is defined by the relative increase in the speed of occurance of disturbance or relative increase in damages or loss. based on this approach the relationship is given as:

$$
G=1-\frac{1}{2} \sum_{m=2}^{N}\left|\frac{\Delta h_{m}}{h_{\max }-h_{\min }}-\frac{\Delta D_{m}}{D_{\max }-D_{\min }}\right|
$$

With:

$G=$ graduality (-), $N=$ number of rainfall events, $\Delta h_{m}=$ change in rainfall depth $(\mathrm{mm})$, $\Delta D_{m}=$ change in damage (M IRRials),$h_{\max }=$ maximum total rainfall depth $(\mathrm{mm})$, $D_{\max }=$ maximum damage $($ M IRRials $), h_{\min }=$ minimum total rainfall depth $(\mathrm{mm})$, $D_{\min }=$ minimum damage $(M$ IRRials $)$

It is now necessary to adopt this relationship for water scarce basins by defining low rainfall depth as:

$$
h_{m_{-} f a \min e}=h_{\text {avg }}-h_{m}
$$

In which $h_{\text {avg }}$ is the minimum fifty year rainfall in $\mathrm{mm}$ and $h_{m \text {-fanine }}$ is the low rainfall depth.

Substitution will result in:

$$
\begin{aligned}
& G=1-\frac{1}{2} \sum_{m=2}^{N}\left|\frac{h_{m}-h_{m-1}}{h_{\max }-h_{\min }}+\frac{D_{m}-D_{m-1}}{D_{\max }-D_{\min }}\right| \\
& D_{m}=2 \times 10^{6} \times\left(\frac{h_{\text {avg }}-h_{m}}{P_{m}}\right)^{0.1681}
\end{aligned}
$$

where, $\mathrm{P}$ is the probability of low rainfall. The damage is determined by a best fit to available damage data as a result of low rainfall and may not be adopted for other basins.

\section{c) Amplitude}

This represents the expected annual damage as:

$$
E A D=\int_{1 / 10000}^{p(D=0)} P D(P) \cdot d P \quad(\text { MIRRials } / \text { year })
$$

where $\mathrm{D}(\mathrm{P})$ is given as the expected damage in the form of a probability function. The relationship may be further simplified to:

$$
E A D=\sum_{i=1}^{n}\left(\Delta P_{i} \times D_{i}\right) \quad \therefore\left\{\begin{array}{l}
D_{i}=\frac{D_{i-1}+D_{i}}{2} \\
\Delta P_{i}=P_{x \geq x}-P_{x \geq x_{i-1}}
\end{array}\right.
$$


where, $\mathrm{n}=$ number of rainfall events, $\mathrm{D}=$ drought damage for section $\mathrm{i}$ ( $M$ IRRials), $\mathrm{P}=$ probability of rainfall occurance for section $\mathrm{i}$ and $\mathrm{EAD}=$ expected annual damage $(\mathrm{M}$ IRRials/year).

\section{CALCULATION OF THE RESILIENCE INDEX}

Using various ranges for the resilience indicators determined from the above, standing at:

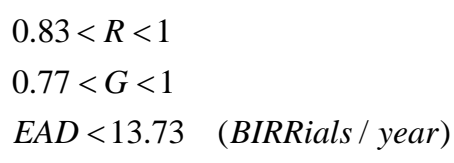

It was observed that the model would produce a relationship for the Lake Urmia's volumes versus varying different figures of reduction in the agriculture allocation up to a figure of $40 \%$. As shown in figure 7 , this relationship is by no means linear, clearly indicating that consideration of resilience indicators will imply a steady and constant rate of change after a breaking point. This indicates that the systems would tend to resist, absorb and recover upon receiving a disturbance. It may be argued here that initially the volume of the lake would increase fairly linraly as reduction in agriculture allocation increases up to a point where the basic characteristics of the system are recovered to its normal condition after reaction. From this point on variations are followed by a near horizontal line indicating further reductions in agriculture allocation would not have any reasonably meaningful effect on the gain in lake volume. In other words, if lake's volume is given first priority in the model, reduction in agriculture allocation would have no effects on the results after a certain (braking) point.

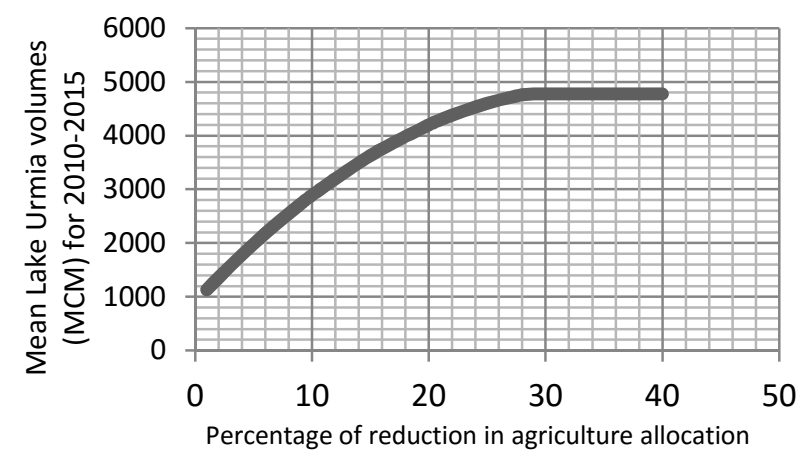

Fig.7. Percentage of reduction in agriculture allocation vs. Lake Urmia volume gains 
It may be deduced that a zone of recovery exists in the resilient attitude in which a system recovers from disturbances (Figure 8). In this case, the more gradual the changes to the reactions to the more suitable the system would perform.

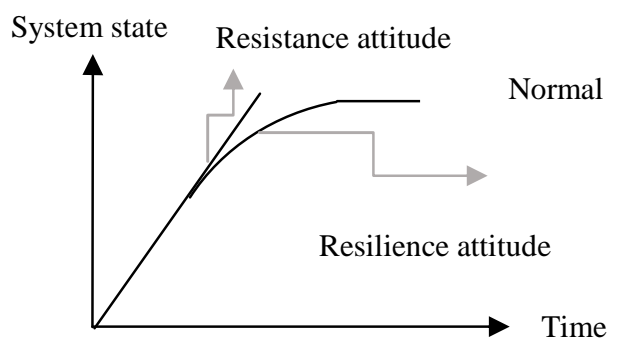

Fig.8. Resilience attitude vs. resistance attitude

In the case of Lake Urmia, it can be seen that the system would regain its normal performance as the graduality and/or the capacity of recovery increase. At the same time a lower amplitude would result in a more suitable outcome for the system. Figure 9 depicts different rates of recovery resulting in different speeds of the system regaining its normal performance. The slope of curve for reaching normal condition is directly proportional to the rate of recovery.

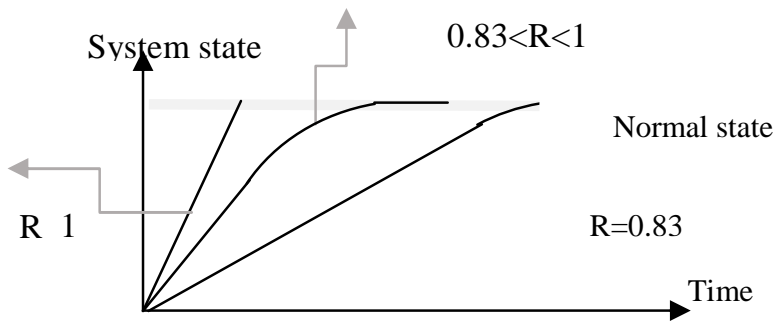

Fig.9. The sensitivity of the system to the rate of recovery

\section{CONCLUSION}

The conventional approach in the management of risks in water scarce basins concentrates on increasing the security of the system when challenged with disturbances. This research has concentrated on identifying the impacts of the disturbances in these basins alongside their security. To achieve this, the sustainability of the system has been considered based on the combination of resistance and resilience approach. A toolbox was developed to adopt a systematic approach to identification and quantification of resilience in water scarce basins. 
Application of the toolbox in the case of Lake Urmia in the Northwest of Iran has enabled accurate assessments of decisions based on conventional approaches. Initially the decision to curb water use for agricultural purposes was investigated. It was shown that if a resilient attitude is adopted the decision to reduce agricultural water allocation by $40 \%$, to regain part of desiccating Lake Urmia's volume, with tremendous social, economic and political impediments may be alleviated to a figure of under $30 \%$. It was argued that the system would initially respond to the changes in agricultural water allocation, however it would remain fairly indifferent once the normal conditions are recovered upon receiving disturbances. By quantifying different resilience indicators for the basin it was shown that resilience is directly related to both recovery rate and graduality while being inversely related to the amplitude.

\section{REFERENCES}

[1] Holling, C. S., Resilience and stability of ecological systems, Annu. Rev. Ecol. Syst. 4, 1$23,1973$.

[2] Adger W. Neil., (2000): Social and ecological resilience: are they related? progress in human geography, doi: 10.1191/030913200701540465.

[3] Walker B., Carpenter S, Anderies J, Abel N, Cumming G. S, Janssen M, Lebel L, Norberg J, Peterson G. D, and Pritchard R., (2002): Resilience management in social-ecological systems: a Working Hypothesis for a Participatory Approach, ecology and society, URL: http://www.consecol.org/vol6/iss1/art14/.

[4] Pimm, S. L., The complexity and stability of ecosystems. Nature 307 (5949), 321-326, 1984.

[5] De Bruijn, K. M., (2004): Resilience and flood risk management, Water Policy. 2004 (6) pp. 53-66.

[6] Yazdandoost, F. and Bozorgy, B., Evaluation of Flood Risk Management Strategies Using Multi-Criteria Analysis, Water Management, Journal of Institution of Civil Engineers, WM5, 261-266, 2008.

[7] Tahmasebi Birgani Y. and Yazdandoost F., A Framework for Evaluating the Persistence in Urban Drainage Risk management Systems, Journal of Hydroenvironment Research, Elsevier, In Press, doi: 10.1016/j.jher, 2014

[8] Tahmasebi Birgani Y., Yazdandoost F. and Moghadam M., (2013): Role of resilience in sustainable urban stromwater management, Hydraulic structures, shahid chamran university, Scientific professional quarterly, http://jhs.scu.ac.ir, 42-50. 
[9] Karamouz, M., Algorithm for Assessment of Water Distribution system's Readiness: Planning for Disasters, Journal of Water Resources Planning and Management, ACSE, PP.244-252, July-August 2009.

[10] Pirniya, Sepideh, (2014): Toolbox Integrated Water Resources Management and uses in the Urmia lake basin, Master Thesis, K. N. Toosi University of Technology.

\section{How to cite this article:}

Yazdandoost F and Moradian S. A resilient approach to integrated water resources management in water scarce basins . J. Fundam. Appl. Sci., 2016, 8(3S), 137-151. 